\title{
DIGITALCOMMONS
}

$11-2014$

\section{Gumbel-Weibull Distribution: Properties and Applications}

\author{
Raid Al-Aqtash \\ Marshall University, alaqtash@marshall.edu \\ Carl Lee \\ Central Michigan University, carl.lee@cmich.edu \\ Felix Famoye \\ Central Michigan University, felix.famoye@cmich.edu
}

Follow this and additional works at: http://digitalcommons.wayne.edu/jmasm

Part of the Applied Statistics Commons, Social and Behavioral Sciences Commons, and the Statistical Theory Commons

\section{Recommended Citation}

Al-Aqtash, Raid; Lee, Carl; and Famoye, Felix (2014) "Gumbel-Weibull Distribution: Properties and Applications," Journal of Modern Applied Statistical Methods: Vol. 13 : Iss. 2 , Article 11.

DOI: $10.22237 /$ jmasm/1414815000

Available at: http://digitalcommons.wayne.edu/jmasm/vol13/iss2/11 


\section{Gumbel-Weibull Distribution: Properties and Applications}

\author{
Raid Al-Aqtash \\ Marshall University \\ Huntington, WV
}

\author{
Carl Lee \\ Central Michigan University \\ Mount Pleasant, MI
}

\author{
Felix Famoye \\ Central Michigan University \\ Mount Pleasant, MI
}

Some properties of the Gumbel-Weibull distribution including the mean deviations and modes are studied. A detailed discussion of regions of unimodality and bimodality is given. The method of maximum likelihood is proposed for estimating the distribution parameters and a simulation is conducted to study the performance of the method. Three tests are given for testing the significance of a distribution parameter. The applications of GumbelWeibull distribution are emphasized. Five data sets are used to illustrate the flexibility of the distribution in fitting unimodal and bimodal data sets.

Keywords: $\quad$ Mean deviation, bimodality, maximum likelihood estimation, lifetime data

\section{Introduction}

Problems on extreme values appeared in the work of Nicholas Bernoulli back in 1709 for studying the problem of the mean largest distance from origin for $n$ random numbers on a straight line (see Johnson et al., 1995, p. 1). During 1920s and 1930s, many papers on the distribution of extremes appeared in the literature. Gumbel (1958) gave detailed results on extreme value theory in his book Statistics of Extremes. Furthermore, Gumbel (1958) has been referred to by Johnson et al. (1995) as the first to bring attention to the possibility of using the Gumbel distribution to model extreme values of random data. For more information on extreme value distributions, see Johnson et al. (1995), Gumbel (1958), Kotz and Nadarajah (2000), and Beirlant et al. (2006).

The Weibull distribution is well known for its ability to model different types of data. Weibull distribution also has many applications in risk analysis and quality

Dr. Al-Aqtash is an Assistant Professor in the Department of Mathematics. Email him at alaqtash@marshall.edu.Dr. Lee is a Professor in the Department of Mathematics. Email him at: carl.lee@cmich.edu.Dr. Famoye is a Professor in Department of Mathematics. Email him at:felix.famoye@cmich.edu. 


\section{GUMBEL-WEIBULL DISTRIBUTION}

control because its hazard rate is decreasing when the shape parameter $a<1$, constant when $a=1$, and increasing when $a>1$. For more information on Weibull distribution, see Johnson et al. (1994).

Alzaatreh, Lee and Famoye (2013) proposed a method for generating new distributions, namely, the $T$ - $X$ family. The cumulative distribution function (CDF) of the $T$-X family is defined as $G(x)=\int_{-\infty}^{W(F(x))} r(t) d t=R(W(F(x)))$, where $X$ is any continuous random variable with $\mathrm{CDF} F(x)$ and probability density function (PDF) $f(x), r(t)$ and $R(t)$ are the PDF and the CDF of a continuous random variable $T$. They further studied a $T-X$ family by defining $W(F(x))=-\log (1-F(x))$, where the random variable $T$ is defined on $(0, \infty)$. Al-Aqtash et al. (2014) defined and studied a family of $T-X$ distributions arising from the logit function $W(F(x))=\ln \{F(x) /(1-F(x))\}$ and provided some general properties of this $T-X$ family, including symmetry, quantile function and Shannon entropy. The CDF of the $T-X$ distribution has the form $G(x)=\int_{-\infty}^{\ln \{F(x) /(1-F(x))\}} r(t) d t=R(\ln \{F(x) /(1-F(x))\})$, where $T$ is defined on $(-\infty, \infty)$. The main difference from the $T$ - $X$ family studied by Alzaatreh, Lee and Famoye (2013) is that the random variable $T$ is defined on $(-\infty, \infty)$ instead of $(0, \infty)$. Taking $T$ to be Gumbel with PDF $r(t)=(1 / \sigma) e^{-(t-v) / \sigma} \exp \left\{-e^{-(t-v) / \sigma}\right\}$ and $X$ to be Weibull with PDF $f(x)=(a / \lambda)(x / \lambda)^{a-1} \exp \left\{-(x / \lambda)^{a}\right\}$, Al-Aqtash et al. (2014) defined the four-parameter Gumbel-Weibull distribution (GWD) with CDF

$$
G(x)=\exp \left\{-e^{\nu / \sigma}\left(e^{(x / \lambda)^{a}}-1\right)^{-1 / \sigma}\right\}=\exp \left\{-\beta\left(e^{(x / \lambda)^{a}}-1\right)^{-1 / \sigma}\right\}
$$

where $0<x<\infty, \beta=e^{v / \sigma}, \sigma, a, \lambda>0$ and $-\infty<v<\infty$. The corresponding PDF is

$$
g(x)=\frac{a \beta}{\lambda \sigma}(x / \lambda)^{a-1} e^{(x / \lambda)^{a}}\left(e^{(x / \lambda)^{a}}-1\right)^{-1-1 / \sigma} \exp \left\{-\beta\left(e^{(x / \lambda)^{a}}-1\right)^{-1 / \sigma}\right\} .
$$

Al-Aqtash et al. (2014) derived some properties of GWD such as moments and Shannon entropy. In this article, additional properties including the mean deviations and modality are studied. In particular, the applications of this distribution are emphasized. 


\section{AL-AQTASH ET AL.}

\section{Mean deviations}

Two measures of spread, namely the mean deviation from the mean and the mean deviation from the median for GWD are now presented. Let $X$ be a random variable from the GWD with mean $\mu$ and median $M$.

The mean deviation from the mean is given by

$$
\begin{aligned}
E(|X-\mu|) & =\int_{0}^{\infty}|x-\mu| g(x) d x=2 \int_{0}^{\mu}(\mu-x) g(x) d x+\int_{0}^{\infty}(x-\mu) g(x) d x \\
& =2 \mu G(\mu)-2 \int_{0}^{\mu} x g(x) d x,
\end{aligned}
$$

where $G(\mu)=\int_{0}^{\mu} g(x) d x$. In a similar way, the mean deviation from the median is given by

$$
\begin{aligned}
E(|X-M|) & =\int_{0}^{\infty}|x-M| g(x) d x \\
& =2 \int_{0}^{M}(M-x) g(x) d x+\int_{0}^{\infty}(x-M) g(x) d x \\
& =\mu-2 \int_{0}^{M} x g(x) d x .
\end{aligned}
$$

The integral $\int_{0}^{v} x g(x) d x$ in (2) and (3) can be computed numerically.

\section{Modes of Gumbel-Weibull distribution}

Al-Aqtash et al. (2014) studied the moments and skewness in detail, and mentioned that GWD can be unimodal or bimodal. However, no study on GWD modes was given. The modes of GWD are provided and the regions of unimodality and bimodality for specific values of $\beta$ are investigated. Differentiating the density of GWD in (1) with respect to $x$ results in

$$
\begin{aligned}
g^{\prime}(x) & =\frac{a \beta}{\lambda^{2} \sigma}\left((x / \lambda)^{a-2} e^{(x / \lambda)^{a}}\left(e^{(x / \lambda)^{a}}-1\right)^{-2-1 / \sigma} \exp \left\{-\beta\left(e^{(x / \lambda)^{a}}-1\right)^{-1 / \sigma}\right\}\right) \\
& \times\left[(a-1)\left(e^{(x / \lambda)^{a}}-1\right)-a(x / \lambda)^{a}+(a / \sigma)(x / \lambda)^{a} e^{(x / \lambda)^{a}}\left\{\beta\left(e^{(x / \lambda)^{a}}-1\right)^{-1 / \sigma}-1\right\}\right] .
\end{aligned}
$$




\section{GUMBEL-WEIBULL DISTRIBUTION}

The derivative $g^{\prime}(x)$ does not exist when $x=0$. Other critical point(s) satisfy $g^{\prime}(x)=0$, hence if there is a mode for GWD, then it will be either at $x=0$ or it will satisfy equation

$$
(a-1)\left(e^{(x / \lambda)^{a}}-1\right)-a(x / \lambda)^{a}+(a / \sigma)(x / \lambda)^{a} e^{(x / \lambda)^{a}}\left\{\beta\left(e^{(x / \lambda)^{a}}-1\right)^{-1 / \sigma}-1\right\}=0 .
$$

In (4), set $\lambda=1$ because $\lambda$ is a scale parameter and it will not affect the distribution shape. The expression on the left hand side of (4) simplifies to

$$
(a-1)\left(e^{x^{a}}-1\right)-a x^{a}+(a / \sigma) x^{a} e^{x^{a}}\left\{\beta\left(e^{x^{a}}-1\right)^{-1 / \sigma}-1\right\} .
$$

Analytical solution of equating (5) to zero is not possible. Numerical approximation is applied to study the modes and the regions of unimodality and bimodality. To study the modes of the GWD, fix $\beta$ and allow $a$ and $\sigma$ to change from 0.01 to 10 at an increment of 0.01 . This gives one million different ordered pairs $(\sigma, a)$. For each ordered pair, (5) is computed as a vector using a vector $x$ with values $0.000001,0.00001,0.0001,0.001$, and then from 0.01 to 10 at an increment of 0.01 . Values of $x$ below 0.000001 cause execution errors as $a$ approaches 10, because $\left(e^{x^{a}}-1\right)^{-1 / \sigma}$ grows rapidly toward $\infty$ as $x$ approaches zero. Because it was observed that the PDF of GWD quickly approaches zero when $x>10, x$ less than 10 was chosen.

A $1000 \times 1000$ matrix $P$ was constructed to store the symbols $\{-2,-1,1,2\}$, and track how many times the sign of the components of the vector (5) changes. If the vector is always negative, set $P_{(\sigma, a)}=-1$ indicating one mode at point $x=0$. If (5) starts negative, then changes to positive and finally becomes negative, set $P_{(\sigma, a)}=-2$ indicating one mode at point $x=0$ and another mode at $x>0$. If (5) starts positive and becomes negative, then changes to positive, and finally becomes negative, set $P_{(\sigma, a)}=2$ indicating two distinct modes at $x>0$. Finally, if (5) starts positive, then becomes negative, set $P_{(\sigma, a)}=1$ indicating one mode at $x>0$.

For fixed $\beta$, the parameter space of GWD is made up of four regions, each region contains a symbol from $\{-1,-2,2,1\}$, separated by boundary curves. The boundary curves are estimated by using regression model. The four distinct regions for GWD modes are marked R1, R2, R3, and R4 corresponding to symbols $-1,-2$, 2 and 1 respectively. The regions $\mathrm{R} 1$ and $\mathrm{R} 4$ determine the region of unimodality, while the regions R2 and R3 determine the region of bimodality. Figures 1, 2 and 


\section{AL-AQTASH ET AL.}

3 show the regions of unimodality and bimodality are not robust to the parameter $\beta$. To save space the following three cases are presented to demonstrate the complexity of the modal property of GWD.

\section{Case $1(\beta=0.5)$}

Figure 1 shows the four distinct regions R1, R2, R3, and R4 for GWD modes, and four PDFs representing the four regions when $\lambda=1$. For example, the PDF of GWD with parameters $\sigma=10$ and $a=1.7$ falls in R2, thus it is bimodal with one mode at 0 and the other mode is at $x=2.71$. In this case, only two curves $\mathrm{C} 1$ and $\mathrm{C} 2$ are found as boundaries for the four regions. The regression models for $\mathrm{C} 1$ and $\mathrm{C} 2$ have $\mathrm{R}^{2}=100 \%$ and are given by

$$
\begin{aligned}
& \mathrm{C} 1: \sigma=-0.04373+5.556 a-0.1453 a^{2}+0.04509 a^{3}, a<1.85, \text { and } \\
& \mathrm{C} 2: \sigma=\left(0.6539-0.5853 a^{-1}+0.2609 a^{-2}-0.2292 a^{-3}\right)^{-2}, 1.52<a<10 .
\end{aligned}
$$
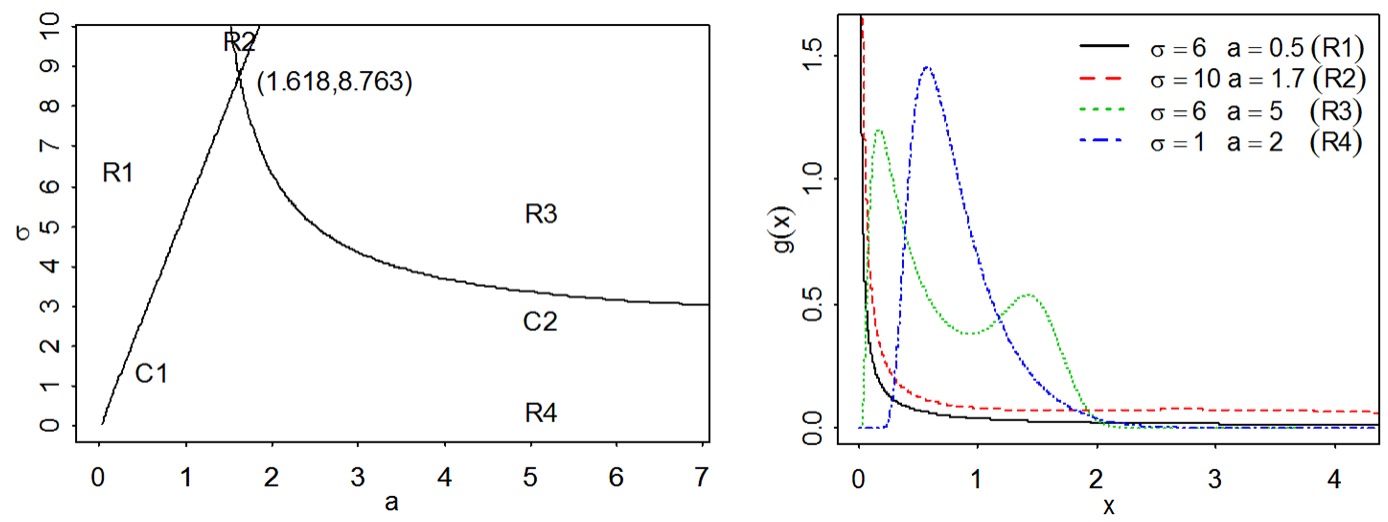

Figure 1. Regions of unimodality and bimodality and PDFs of GWD when $\beta=0.5$ and $\lambda=1$

\section{Case $2(\beta=1)$}

Figure 2 shows the four distinct regions R1, R2, R3, and R4 for GWD modes, and four PDFs from the four regions when $\lambda=1$. For example, the PDF corresponding to $\sigma=10$ and $a=1.4$ is bimodal, the first mode is at 0 and the other mode is at $x=3.55$. Three curves $\mathrm{C} 1, \mathrm{C} 2$, and $\mathrm{C} 3$ separate the four regions. The regression models for $\mathrm{C} 1, \mathrm{C} 2$, and $\mathrm{C} 3$ all have $\mathrm{R}^{2}=100 \%$ and are given by 


\section{GUMBEL-WEIBULL DISTRIBUTION}

$\mathrm{C} 1: \sigma=-0.05499+6.977 a-0.2862 a^{2}+0.1072 a^{3}, a<1.49$,

C2: $\sigma=\left(-1.668+2.999 a^{1 / 2}-1.655 a+0.3443 a^{3 / 2}\right)^{-1}, 1.27<a<3.26$, and

C3: $\sigma=-12.26+14.43 a^{1 / 2}-4.332 a+0.5391 a^{3 / 2}, 3.26<a<10$.
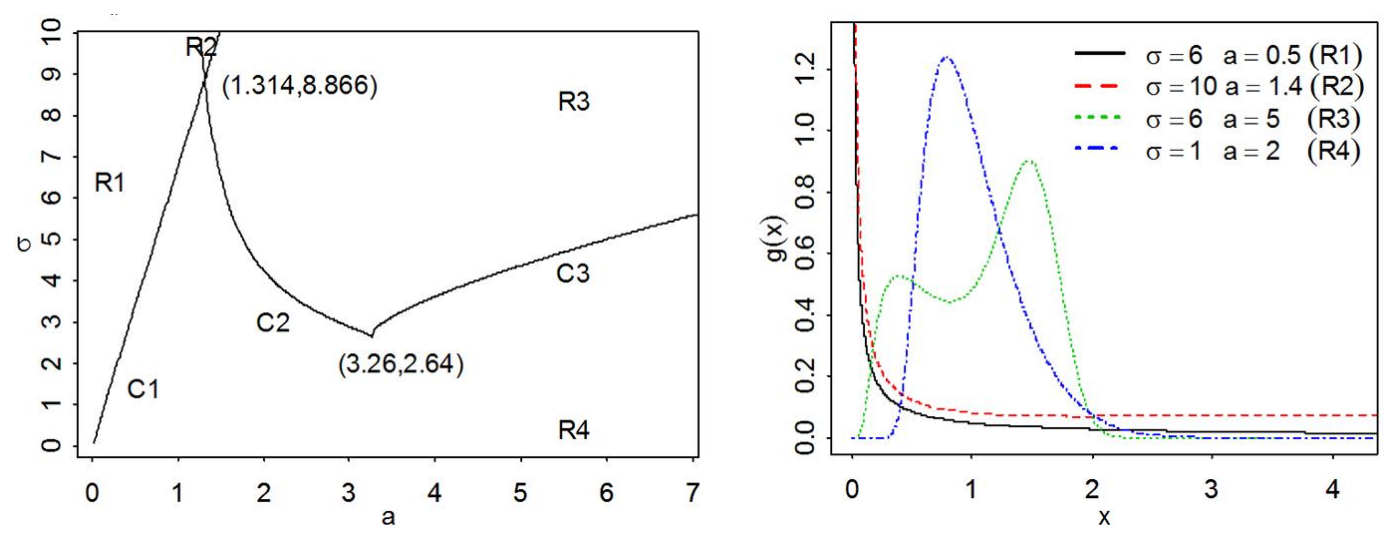

Figure 2. Regions of unimodality and bimodality and PDFs of GWD when $\beta=1$ and $\lambda=1$

\section{Case $3(\beta=2)$}

Figure 3 shows the four distinct regions R1, R2, R3, and R4 for GWD modes separated by three curves and four PDFs from the four regions when $\lambda=1$. For example, the PDF corresponding to $\sigma=10$ and $a=1$ is bimodal with modes at 0 and at $x=6.83$. The three regression models, all with $\mathrm{R}^{2}=100 \%$, are given by

$\mathrm{C} 1: \sigma=\left(-0.01444+25.78 a^{3 / 2}+0.1418 a^{3}-0.1129 a^{9 / 2}\right)^{2 / 3}, a<1.15$,

C2: $\sigma=\left(-0.9390+2.688 a^{2}-1.814 a^{4}+0.4480 a^{6}\right)^{-2}, 0.93<a<1.289$, and

C3: $\sigma=0.3479+1.531 a-0.1226 a^{2}+0.004977 a^{3}, 1.289<a<5.05$. 


\section{AL-AQTASH ET AL.}
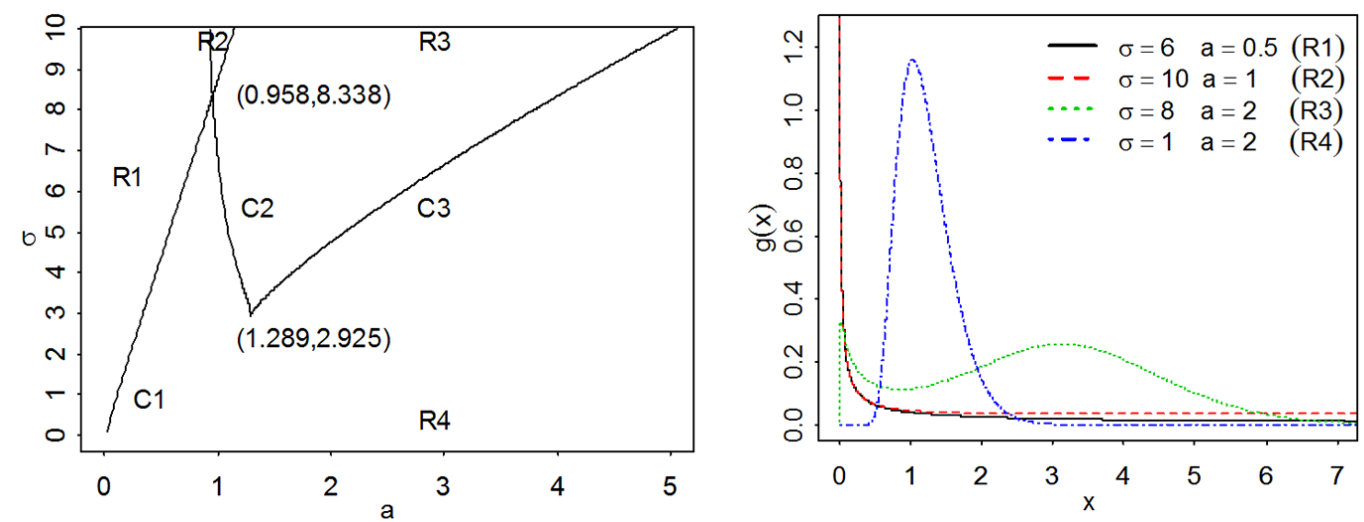

Figure 3. Regions of unimodality and bimodality and PDFs of GWD when $\beta=2$ and $\lambda=1$

The distance between the two modes when GWD is bimodal was also examined, which is demonstrated in Table 1. From Table 1 it is observed that, when $\sigma$ increases and all the other parameters are fixed, the distance between two modes increases, and when $a$ increases, the distance decreases. However, no clear increasing or decreasing pattern is observed as $\beta$ increases when all other parameters are fixed. Figure 4 further illustrates the diverse shapes of GWD and the distances between two modes when $\sigma, \beta$, or $a$ changes, respectively.
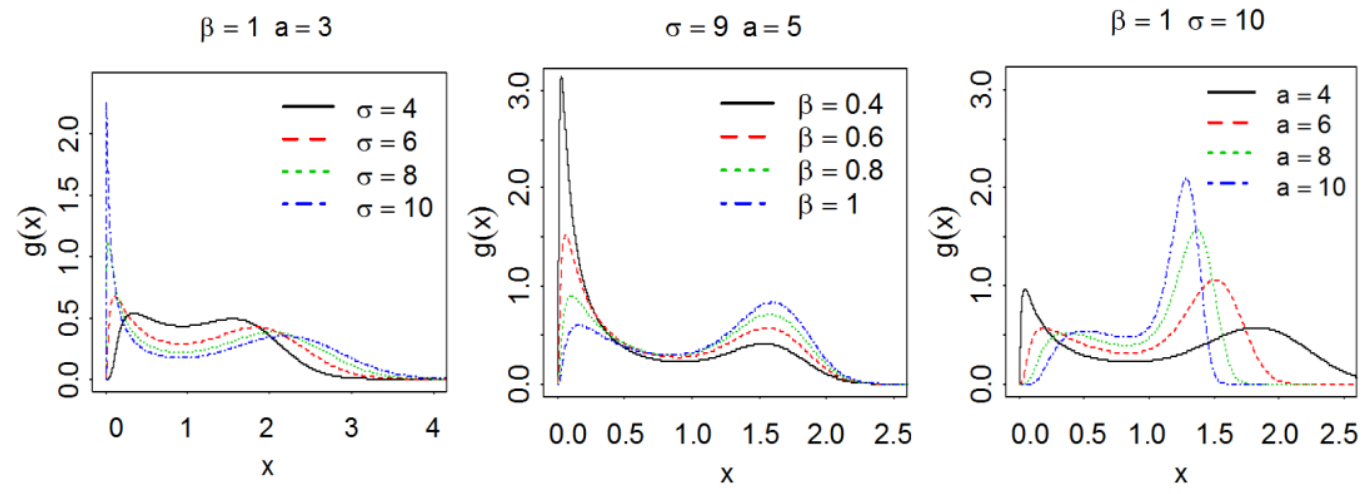

Figure 4. PDFs of GWD when the distribution is bimodal and $\lambda=1$ 


\section{GUMBEL-WEIBULL DISTRIBUTION}

Table 1. Distance between two modes when GWD is bimodal and $\lambda=1$

\begin{tabular}{|c|c|c|c|c|c|c|}
\hline$a$ & $\sigma$ & $\beta=0.6$ & $\beta=0.8$ & $\beta=1$ & $\beta=1.5$ & $\beta=2$ \\
\hline \multirow{7}{*}{2} & 4 & & & & 1.68 & \\
\hline & 5 & & 1.62 & 1.91 & 2.13 & 2.14 \\
\hline & 6 & 1.82 & 2.07 & 2.21 & 2.44 & 2.57 \\
\hline & 7 & 2.14 & 2.29 & 2.43 & 2.68 & 2.87 \\
\hline & 8 & 2.34 & 2.49 & 2.61 & 2.89 & 3.10 \\
\hline & 9 & 2.51 & 2.65 & 2.79 & 3.07 & 3.30 \\
\hline & 10 & 2.65 & 2.80 & 2.94 & 3.25 & 3.49 \\
\hline \multirow{7}{*}{3} & 4 & & 1.23 & 1.20 & & \\
\hline & 5 & 1.50 & 1.52 & 1.51 & 1.35 & \\
\hline & 6 & 1.68 & 1.70 & 1.71 & 1.67 & \\
\hline & 7 & 1.81 & 1.85 & 1.87 & 1.88 & 1.78 \\
\hline & 8 & 1.91 & 1.96 & 1.99 & 2.03 & 2.01 \\
\hline & 9 & 1.99 & 2.05 & 2.09 & 2.16 & 2.18 \\
\hline & 10 & 2.06 & 2.12 & 2.17 & 2.27 & 2.31 \\
\hline \multirow{7}{*}{4} & 4 & 1.04 & 0.98 & 0.87 & & \\
\hline & 5 & 1.27 & 1.22 & 1.15 & & \\
\hline & 6 & 1.43 & 1.39 & 1.35 & & \\
\hline & 7 & 1.55 & 1.53 & 1.50 & 1.37 & \\
\hline & 8 & 1.62 & 1.62 & 1.61 & 1.53 & \\
\hline & 9 & 1.69 & 1.71 & 1.70 & 1.67 & 1.54 \\
\hline & 10 & 1.75 & 1.77 & 1.78 & 1.77 & 1.69 \\
\hline \multirow{7}{*}{5} & 4 & 0.87 & 0.78 & & & \\
\hline & 5 & 1.07 & 0.99 & 0.88 & & \\
\hline & 6 & 1.22 & 1.16 & 1.08 & & \\
\hline & 7 & 1.33 & 1.29 & 1.23 & & \\
\hline & 8 & 1.43 & 1.39 & 1.35 & 1.17 & \\
\hline & 9 & 1.49 & 1.47 & 1.44 & 1.32 & \\
\hline & 10 & 1.55 & 1.54 & 1.52 & 1.42 & 1.21 \\
\hline \multirow{7}{*}{6} & 4 & 0.73 & 0.62 & & & \\
\hline & 5 & 0.92 & 0.81 & & & \\
\hline & 6 & 1.05 & 0.97 & 0.87 & & \\
\hline & 7 & 1.17 & 1.09 & 1.02 & & \\
\hline & 8 & 1.26 & 1.20 & 1.13 & & \\
\hline & 9 & 1.34 & 1.29 & 1.23 & 1.03 & \\
\hline & 10 & 1.40 & 1.36 & 1.32 & 1.16 & \\
\hline
\end{tabular}




\section{AL-AQTASH ET AL.}

\section{Parameter estimation}

Next, the parameter estimation using the maximum likelihood method is addressed

and a simulation is conducted to study the performance of the method. Let $\left(x_{1}, x_{2}\right.$ $\ldots x_{n}$ ) be a random sample from a GWD with parameters $\beta, \sigma, a$, and $\lambda$, then the log-likelihood function from (1) is given by

$$
\begin{aligned}
\ell(\beta, \sigma, a, \lambda)= & \ell=\sum_{i=1}^{n} \ln \left(g\left(x_{i}\right)\right) \\
= & n(\ln \beta+\ln a-\ln \sigma-a \ln \lambda)+(a-1) \sum_{i=1}^{n} \ln x_{i}-\frac{1}{\sigma} \sum_{i=1}^{n}\left(\frac{x_{i}}{\lambda}\right)^{a} \\
& -\left(1+\frac{1}{\sigma}\right) \sum_{i=1}^{n} \ln \left\{1-e^{-\left(x_{i} / \lambda\right)^{a}}\right\}-\beta \sum_{i=1}^{n}\left\{e^{(x / \lambda)^{a}}-1\right\}^{-1 / \sigma} .
\end{aligned}
$$

The first partial derivatives of (6) are

$$
\begin{aligned}
\frac{\partial \ell}{\partial \beta}= & \frac{n}{\beta}-\sum_{i=1}^{n}\left\{e^{\left(x_{i} / \lambda\right)^{a}}-1\right\}^{-1 / \sigma}, \\
\frac{\partial \ell}{\partial \sigma}= & -\frac{n}{\sigma}+\frac{1}{\sigma^{2}} \sum_{i=1}^{n}\left(x_{i} / \lambda\right)^{a}+\frac{1}{\sigma^{2}} \sum_{i=1}^{n} \ln \left\{1-e^{-\left(x_{i} / \lambda\right)^{a}}\right\} \\
& -\frac{\beta}{\sigma^{2}} \sum_{i=1}^{n}\left\{e^{\left(x_{i} / \lambda\right)^{a}}-1\right\}^{-1 / \sigma} \ln \left\{e^{\left(x_{i} / \lambda\right)^{a}}-1\right\}, \\
\frac{\partial \ell}{\partial a}= & \frac{n}{a}-n \ln \lambda+\sum_{i=1}^{n} \ln x_{i}-\frac{1}{\sigma} \sum_{i=1}^{n}\left(x_{i} / \lambda\right)^{a} \ln \left(x_{i} / \lambda\right) \\
& -(1+1 / \sigma) \sum_{i=1}^{n}\left(x_{i} / \lambda\right)^{a} \ln \left(x_{i} / \lambda\right)\left\{e^{\left(x_{i} / \lambda\right)^{a}}-1\right\}^{-1} \\
& +\frac{\beta}{\sigma} \sum_{i=1}^{n}\left\{e^{\left(x_{i} / \lambda\right)^{a}}-1\right\}^{-1-1 / \sigma} e^{\left(x_{i} / \lambda\right)^{a}}\left(x_{i} / \lambda\right)^{a} \ln \left(x_{i} / \lambda\right),
\end{aligned}
$$

and 


\section{GUMBEL-WEIBULL DISTRIBUTION}

$$
\begin{aligned}
\frac{\partial \ell}{\partial \lambda} & =-\frac{n a}{\lambda}+\frac{a}{\lambda \sigma} \sum_{i=1}^{n}\left(x_{i} / \lambda\right)^{a}+\frac{a}{\lambda}(1+1 / \sigma) \sum_{i=1}^{n}\left(x_{i} / \lambda\right)^{a}\left\{e^{\left(x_{i} / \lambda\right)^{a}}-1\right\}^{-1} \\
& -\frac{a \beta}{\lambda \sigma} \sum_{i=1}^{n}\left(x_{i} / \lambda\right)^{a} e^{\left(x_{i} / \lambda\right)^{a}}\left\{e^{\left(x_{i} / \lambda\right)^{a}}-1\right\}^{-1-1 / \sigma} .
\end{aligned}
$$

On setting (7) through (10) equal to zero and solving the system of equations iteratively using NLMIXED procedure in SAS, the maximum likelihood estimates of the parameters are obtained.

Because the parameters $a$ and $\lambda$ are from Weibull distribution (WD), and the parameters $\beta$ and $\sigma$ are from Gumbel distribution (GD), moment estimates of WD parameters and GD parameters are used as initial estimates. By assuming that $\left(x_{1}, x_{2}\right.$ $\left.\ldots x_{n}\right)$ has WD, $w_{i}=\ln \left(x_{i}\right)$ can be computed. The initial estimates for $a$ and $\lambda$ are $a_{0}=\pi /\left(s_{w} \sqrt{6}\right)$ and $\lambda_{0}=\exp \left(\bar{w}-\gamma / a_{0}\right)$, where $\bar{w}$ and $s_{w}$ are respectively the mean and the standard deviation of the sample $\left(w_{1} \ldots w_{n}\right)$ (Johnson et al., 1994, pp. $635-643)$, and $\gamma=-\Gamma^{\prime}(1) \approx 0.57722$ is Euler's constant. The random sample $\left(x_{1}, x_{2}\right.$ $\left.\ldots x_{n}\right)$ is transformed to a sample from GD by $z_{i}=\ln \left(\exp \left(x_{i} / \lambda_{0}\right)^{a_{0}}-1\right)$. The initial

estimates for $\beta$ and $\sigma$ are $\beta_{0}=e^{v_{0} / \sigma_{0}}$ and $\sigma_{0}=s_{z} \sqrt{6} / \pi$, where $v_{0}=\bar{z}-\gamma \sigma_{0}$ and $\sigma_{0}$ are the moment estimates from GD, $\bar{z}$ and $s_{z}$ are respectively the mean and standard deviation of $\left(z_{1} \ldots z_{n}\right)$ (Johnson et al., 1995, p. 12).

A simulation study is conducted to examine the performance of the maximum likelihood estimates (MLEs). The bias (actual - estimate) and standard deviation are used to measure the performance. Three sample sizes $n=250,500$ and 1000 are used. The data are simulated from standard exponential distribution and then transformed into GWD using the transformation $X=\lambda\left(\ln \left\{(Y / \beta)^{-\sigma}+1\right\}\right)^{1 / a}$. The process is repeated 100 times for each parameter combination and each sample size. This simulation study is conducted for many parameter combinations, but for brevity the results for the parameter combinations $\beta=0.5,1,4, \sigma=2, a=1,2,4$, and $\lambda=1$ are reported.

The average bias and the standard deviation of the MLEs are computed and presented in Table 2. From the table it is observed that the bias appears reasonable and in general the standard deviation decreases as $n$ increases. These observations also hold for the other parameter combinations that are not reported in Table 2. The simulation study shows that the maximum likelihood method is appropriate for estimating the GWD parameters. 


\section{AL-AQTASH ET AL.}

Table 2. Average bias and standard deviation for MLEs when $\sigma=2$ and $\lambda=1$

\begin{tabular}{|c|c|c|c|c|c|c|c|c|c|c|}
\hline \multirow[b]{2}{*}{$\beta$} & \multirow[b]{2}{*}{$a$} & \multirow[b]{2}{*}{$n$} & \multicolumn{2}{|c|}{$\hat{\beta}$} & \multicolumn{2}{|c|}{$\hat{\sigma}$} & \multicolumn{2}{|c|}{$\hat{a}$} & \multicolumn{2}{|c|}{$\lambda$} \\
\hline & & & bias & sd & bias & sd & bias & sd & bias & sd \\
\hline \multirow{9}{*}{0.5} & & 250 & -0.0096 & 0.0867 & -0.0354 & 0.1514 & -0.0734 & 0.2750 & -0.0307 & 0.2047 \\
\hline & 1 & 500 & 0.0082 & 0.0830 & -0.0473 & 0.1326 & -0.0853 & 0.2192 & -0.0574 & 0.1931 \\
\hline & & 1000 & 0.0097 & 0.0642 & -0.0329 & 0.1003 & -0.0532 & 0.1565 & -0.0471 & 0.1482 \\
\hline & & 250 & 0.0065 & 0.0942 & -0.1094 & 0.3163 & -0.0893 & 0.2785 & -0.0336 & 0.1140 \\
\hline & 2 & 500 & 0.0136 & 0.0879 & -0.1103 & 0.2745 & -0.0921 & 0.2170 & -0.0341 & 0.1054 \\
\hline & & 1000 & 0.0110 & 0.0652 & -0.0674 & 0.2015 & -0.0532 & 0.1565 & -0.0230 & 0.0754 \\
\hline & & 250 & 0.0065 & 0.0942 & -0.2188 & 0.6325 & -0.0893 & 0.2785 & -0.0151 & 0.0562 \\
\hline & 4 & 500 & 0.0148 & 0.0874 & -0.2270 & 0.5470 & -0.0935 & 0.2162 & -0.0156 & 0.0512 \\
\hline & & 1000 & 0.0114 & 0.0650 & -0.1363 & 0.4031 & -0.0539 & 0.1570 & -0.0109 & 0.0372 \\
\hline \multirow{9}{*}{1} & & 250 & -0.0049 & 0.1832 & -0.0319 & 0.1281 & -0.0644 & 0.2429 & -0.0380 & 0.2055 \\
\hline & 1 & 500 & 0.0443 & 0.1597 & -0.0413 & 0.1030 & -0.0482 & 0.1738 & -0.0862 & 0.1872 \\
\hline & & 1000 & 0.0346 & 0.1375 & -0.0300 & 0.0893 & -0.0317 & 0.1248 & -0.0625 & 0.1626 \\
\hline & & 250 & 0.0335 & 0.2084 & -0.1343 & 0.3083 & -0.0905 & 0.2539 & -0.0462 & 0.1276 \\
\hline & 2 & 500 & 0.0663 & 0.1723 & -0.1317 & 0.2518 & -0.0755 & 0.1812 & -0.0550 & 0.1061 \\
\hline & & 1000 & 0.0426 & 0.1435 & -0.0790 & 0.1956 & -0.0431 & 0.1287 & -0.0348 & 0.0845 \\
\hline & & 250 & 0.0335 & 0.2084 & -0.2685 & 0.6166 & -0.0905 & 0.2539 & -0.0210 & 0.0625 \\
\hline & 4 & 500 & 0.0682 & 0.1715 & -0.2591 & 0.4872 & -0.0722 & 0.1788 & -0.0263 & 0.0512 \\
\hline & & 1000 & 0.0415 & 0.1431 & -0.1498 & 0.3893 & -0.0410 & 0.1296 & -0.0160 & 0.0416 \\
\hline \multirow{9}{*}{4} & & 250 & -0.0876 & 0.7596 & -0.0017 & 0.0956 & -0.1131 & 0.4299 & 0.0160 & 0.2424 \\
\hline & 1 & 500 & 0.0049 & 0.6856 & -0.0160 & 0.0792 & -0.0755 & 0.2637 & -0.0192 & 0.2154 \\
\hline & & 1000 & -0.0265 & 0.7518 & -0.0021 & 0.0774 & -0.0249 & 0.2223 & -0.0195 & 0.2401 \\
\hline & & 250 & 0.3277 & 0.8897 & -0.1143 & 0.2423 & -0.0298 & 0.3595 & -0.0722 & 0.1654 \\
\hline & 2 & 500 & 0.3395 & 0.8917 & -0.1004 & 0.2081 & 0.0101 & 0.2951 & -0.0761 & 0.1637 \\
\hline & & 1000 & 0.3088 & 0.8031 & -0.0810 & 0.1858 & 0.0276 & 0.2184 & -0.0657 & 0.1430 \\
\hline & & 250 & 0.3712 & 0.8546 & -0.1404 & 0.4622 & -0.0696 & 0.4325 & -0.0225 & 0.0836 \\
\hline & 4 & 500 & 0.3876 & 0.8298 & -0.1720 & 0.3838 & -0.0244 & 0.2715 & -0.0283 & 0.0754 \\
\hline & & 1000 & 0.2294 & 0.8272 & -0.1171 & 0.3705 & -0.0106 & 0.2208 & -0.0189 & 0.0720 \\
\hline
\end{tabular}

\section{Tests about parameter $\beta$}

When $\beta=1$, the GWD reduces to

$$
g_{0}(x)=\frac{a}{\lambda \sigma}(x / \lambda)^{a-1} e^{(x / \lambda)^{a}}\left(e^{(x / \lambda)^{a}}-1\right)^{-1-1 / \sigma} \exp \left\{-\left(e^{(x / \lambda)^{a}}-1\right)^{-1 / \sigma}\right\} .
$$

The three-parameter GWD in (11) can be compared with the four-parameter GWD in (1) by testing the null hypothesis

$$
H_{0}: \beta=1 \text { against } H_{1}: \beta \neq 1 \text {. }
$$




\section{GUMBEL-WEIBULL DISTRIBUTION}

The hypotheses in (12) are tested by using the likelihood ratio test (Neyman and Pearson, 1928), the Wald test (Wald, 1943), or the score test (Rao, 1948).

The likelihood ratio statistic for testing (12) is $\eta=-2\left[\ell_{0}(\tilde{\sigma}, \tilde{a}, \tilde{\lambda})-\ell_{1}(\hat{\beta}, \hat{\sigma}, \hat{a}, \hat{\lambda})\right]$, where $\ell_{0}(\tilde{\sigma}, \tilde{a}, \tilde{\lambda})$ is the log-likelihood value of the three-parameter GWD and $\ell_{1}(\hat{\beta}, \hat{\sigma}, \hat{a}, \hat{\lambda})$ is the log-likelihood value of the four-parameter GWD. The likelihood ratio statistic $\eta$ has asymptotic chi-square distribution with one degree of freedom.

The Wald statistic for testing (12) is $Z_{\hat{\beta}}=(\hat{\beta}-1) / S E_{\hat{\beta}}$, where $\hat{\beta}$ is the MLE from the four-parameter GWD and $S E_{\hat{\beta}}$ is the standard error of $\hat{\beta}$. The Wald statistic $Z_{\hat{\beta}}$ has an approximate standard normal distribution.

The score statistic for testing (12) is $S=V^{T} I^{-1} V$, where the score vector $V$ is the $4 \times 1$ gradient vector of $\ell$ with entries $V_{i}=\partial \ell / \partial \theta_{i}, I$ is the $4 \times 4$ information matrix with entries $I_{i, j}=-E\left[\partial^{2} \ell / \partial \theta_{i} \partial \theta_{j}\right], \ell$ is the GWD log-likelihood function in (6), $\theta_{1}=\beta, \theta_{2}=\sigma, \theta_{3}=a$, and $\theta_{4}=\lambda$, and $S$ is computed under the null hypothesis $\beta=1$. The score statistic $S$ has an approximate chi-square distribution with one degree of freedom.

A simulation study is conducted to compare the powers of the three tests for parameter $\beta$. Three sample sizes $n=250,500$ and 1000 are used in the study. The data are simulated from GWD as described under Parameter Estimation, and the parameters are estimated by the method of maximum likelihood. The simulation is repeated 200 times for each parameter combination and each sample size. The proportion of times of rejecting $H_{0}$ is used to estimate the power of each test. Two significance levels, 5\% and 10\%, are used and the results are similar. Many parameter combinations are used in the simulation. For brevity, Table 3 reports the results for $5 \%$ and the parameter values $\beta=0.5,1,2,4, \sigma=1.6,2, a=1,2,3$, 4, and $\lambda=1$.

When $\beta \leq 1$, it is observed from Table 3 that the score test is the most powerful followed by the Wald test while the likelihood ratio test is poor. On the contrary, when $\beta>1$, the likelihood ratio test is the most powerful followed by the score test and the Wald test. In general, the power increases as the sample size increases. 
AL-AQTASH ET AL.

Table 3. The proportion of times the null hypothesis is rejected when $\lambda=1$

\begin{tabular}{|c|c|c|c|c|c|c|c|c|c|c|c|}
\hline \multirow[b]{2}{*}{$\sigma$} & \multirow[b]{2}{*}{$\beta$} & \multirow[b]{2}{*}{$a$} & \multicolumn{3}{|c|}{$n=250$} & \multicolumn{3}{|c|}{$n=500$} & \multicolumn{3}{|c|}{$n=1000$} \\
\hline & & & LR & Wald & Score & LR & Wald & Score & LR & Wald & Score \\
\hline \multirow{16}{*}{1.6} & \multirow{4}{*}{0.5} & 1 & 0.000 & 0.005 & 0.430 & 0.000 & 0.045 & 0.745 & 0.020 & 0.600 & 0.955 \\
\hline & & 2 & 0.000 & 0.000 & 0.420 & 0.000 & 0.060 & 0.685 & 0.040 & 0.600 & 0.940 \\
\hline & & 3 & 0.000 & 0.005 & 0.435 & 0.000 & 0.060 & 0.650 & 0.010 & 0.530 & 0.945 \\
\hline & & 4 & 0.000 & 0.000 & 0.495 & 0.000 & 0.060 & 0.690 & 0.020 & 0.620 & 0.970 \\
\hline & \multirow{4}{*}{1} & 1 & 0.000 & 0.000 & 0.015 & 0.000 & 0.005 & 0.050 & 0.005 & 0.040 & 0.070 \\
\hline & & 2 & 0.000 & 0.000 & 0.060 & 0.000 & 0.060 & 0.165 & 0.015 & 0.125 & 0.145 \\
\hline & & 3 & 0.000 & 0.000 & 0.070 & 0.000 & 0.060 & 0.140 & 0.010 & 0.060 & 0.095 \\
\hline & & 4 & 0.000 & 0.000 & 0.070 & 0.000 & 0.045 & 0.120 & 0.015 & 0.055 & 0.080 \\
\hline & \multirow{4}{*}{2} & 1 & 0.090 & 0.000 & 0.005 & 0.340 & 0.010 & 0.020 & 0.740 & 0.110 & 0.405 \\
\hline & & 2 & 0.070 & 0.000 & 0.005 & 0.220 & 0.000 & 0.020 & 0.720 & 0.055 & 0.340 \\
\hline & & 3 & 0.070 & 0.000 & 0.000 & 0.315 & 0.005 & 0.050 & 0.670 & 0.095 & 0.395 \\
\hline & & 4 & 0.065 & 0.000 & 0.005 & 0.295 & 0.000 & 0.030 & 0.675 & 0.115 & 0.350 \\
\hline & \multirow{4}{*}{4} & 1 & 0.605 & 0.010 & 0.120 & 0.980 & 0.050 & 0.450 & 1.000 & 0.515 & 0.975 \\
\hline & & 2 & 0.585 & 0.015 & 0.075 & 0.935 & 0.040 & 0.435 & 1.000 & 0.400 & 0.945 \\
\hline & & 3 & 0.490 & 0.000 & 0.065 & 0.935 & 0.025 & 0.385 & 1.000 & 0.410 & 0.945 \\
\hline & & 4 & 0.650 & 0.030 & 0.100 & 0.935 & 0.075 & 0.475 & 1.000 & 0.585 & 0.980 \\
\hline \multirow{16}{*}{2} & \multirow{4}{*}{0.5} & 1 & 0.000 & 0.090 & 0.660 & 0.030 & 0.560 & 0.945 & 0.480 & 0.970 & 1.000 \\
\hline & & 2 & 0.000 & 0.085 & 0.715 & 0.020 & 0.495 & 0.925 & 0.545 & 0.990 & 1.000 \\
\hline & & 3 & 0.000 & 0.080 & 0.740 & 0.035 & 0.490 & 0.940 & 0.525 & 0.985 & 1.000 \\
\hline & & 4 & 0.005 & 0.120 & 0.745 & 0.025 & 0.575 & 0.960 & 0.545 & 0.985 & 1.000 \\
\hline & \multirow{4}{*}{1} & 1 & 0.000 & 0.000 & 0.050 & 0.000 & 0.000 & 0.060 & 0.005 & 0.030 & 0.090 \\
\hline & & 2 & 0.000 & 0.020 & 0.100 & 0.010 & 0.050 & 0.110 & 0.030 & 0.070 & 0.080 \\
\hline & & 3 & 0.000 & 0.000 & 0.085 & 0.000 & 0.065 & 0.105 & 0.025 & 0.045 & 0.055 \\
\hline & & 4 & 0.000 & 0.010 & 0.090 & 0.005 & 0.065 & 0.080 & 0.010 & 0.025 & 0.030 \\
\hline & \multirow{4}{*}{2} & 1 & 0.360 & 0.005 & 0.030 & 0.730 & 0.080 & 0.360 & 0.985 & 0.760 & 0.925 \\
\hline & & 2 & 0.345 & 0.010 & 0.060 & 0.655 & 0.130 & 0.345 & 0.920 & 0.715 & 0.820 \\
\hline & & 3 & 0.290 & 0.005 & 0.055 & 0.645 & 0.105 & 0.360 & 0.940 & 0.735 & 0.830 \\
\hline & & 4 & 0.290 & 0.005 & 0.040 & 0.715 & 0.115 & 0.370 & 0.930 & 0.775 & 0.860 \\
\hline & \multirow{4}{*}{4} & 1 & 0.940 & 0.160 & 0.425 & 1.000 & 0.515 & 0.935 & 1.000 & 1.000 & 1.000 \\
\hline & & 2 & 0.840 & 0.110 & 0.285 & 1.000 & 0.380 & 0.835 & 1.000 & 0.955 & 1.000 \\
\hline & & 3 & 0.875 & 0.100 & 0.305 & 1.000 & 0.485 & 0.885 & 1.000 & 0.940 & 1.000 \\
\hline & & 4 & 0.880 & 0.095 & 0.365 & 1.000 & 0.510 & 0.905 & 1.000 & 0.965 & 1.000 \\
\hline
\end{tabular}

\section{Applications}

Applications of the GWD to five data sets are now presented. These five data sets exhibit various shapes of distribution including right-skewed, approximately symmetric, left-skewed, reversed J-shape and bimodal distributions for demonstrating the flexibility of the GWD for fitting real world data. The parameters are estimated by using the method of maximum likelihood. The fit is compared to 


\section{GUMBEL-WEIBULL DISTRIBUTION}

other distributions based on the p-value of the Kolmogorov-Smirnov (K-S) statistic, and the Akaike information criterion (AIC).

\section{Breaking stress of carbon fibers data}

The data for breaking stress of carbon fibers of $50 \mathrm{~mm}$ length (GPa) in Table 4 is obtained from Nicholas and Padgett (2006). This data was used by Cordeiro and Lemonte (2011) to illustrate the application of the four-parameter beta-BirnbaumSaunders distribution (BBS) when compared to the two-parameter BirnbaumSaunders distribution (Birnbaum and Saunders, 1969). The data set is unimodal and is approximately symmetric (skewness $=-0.13$ and kurtosis $=0.34$ ).

Table 4. Breaking stress of carbon fibers data

\begin{tabular}{lllllllllll}
\hline 0.39 & 0.85 & 1.08 & 1.25 & 1.47 & 1.57 & 1.61 & 1.61 & 1.69 & 1.80 & 1.84 \\
1.87 & 1.89 & 2.03 & 2.03 & 2.05 & 2.12 & 2.35 & 2.41 & 2.43 & 2.48 & 2.50 \\
2.53 & 2.55 & 2.55 & 2.56 & 2.59 & 2.67 & 2.73 & 2.74 & 2.79 & 2.81 & 2.82 \\
2.85 & 2.87 & 2.88 & 2.93 & 2.95 & 2.96 & 2.97 & 3.09 & 3.11 & 3.11 & 3.15 \\
3.15 & 3.19 & 3.22 & 3.22 & 3.27 & 3.28 & 3.31 & 3.31 & 3.33 & 3.39 & 3.39 \\
3.56 & 3.60 & 3.65 & 3.68 & 3.70 & 3.75 & 4.20 & 4.38 & 4.42 & 4.70 & 4.90 \\
\hline
\end{tabular}

Table 5. MLEs for breaking stress of carbon fibers data (standard errors in parentheses)

\begin{tabular}{lcccc} 
Distribution & \multicolumn{1}{c}{ BBS $^{*}$} & BED & BGE & GWD \\
\hline & $\hat{a}=0.1930$ & $\hat{a}=7.5072$ & $\hat{a}=0.6473$ & $\hat{\beta}=3.4359$ \\
& $(0.0259)$ & $(0.7642)$ & $(0.3077)$ & $(1.1494)$ \\
& $\hat{b}=1876.7324$ & $\hat{b}=20.9967$ & $\hat{b}=1198.50$ & $\hat{\sigma}=5.5673$ \\
Parameter & $(605.05)$ & $(1.4865)$ & $(5.9057)$ & $(2.8064)$ \\
estimates & $\hat{\alpha}=1.0445$ & $\hat{\lambda}=0.1131$ & $\hat{\alpha}=5.099$ & $\hat{a}=2.4231$ \\
& $(0.0036)$ & $(0.0170)$ & $(1.9670)$ & $(0.5078)$ \\
& $\hat{\beta}=57.6001$ & & & \\
& $(0.3313)$ & & $\hat{\lambda}=0.0824$ & $\hat{\lambda}=1.1324$ \\
& -91.36 & -91.22 & -85.96 & -84.83 \\
\hline Log Likelihood & 190.7 & 188.4 & 179.9 & 177.7 \\
\hline AIC & 0.1422 & 0.1338 & 0.0817 & 0.0666 \\
\hline K-S & 0.1384 & 0.1884 & 0.7710 & 0.9313 \\
\hline p-value & $\hat{y}$ & & & \\
\hline
\end{tabular}

*Parameter estimates and value of AIC statistic from Cordeiro and Lemonte (2011). 


\section{AL-AQTASH ET AL.}

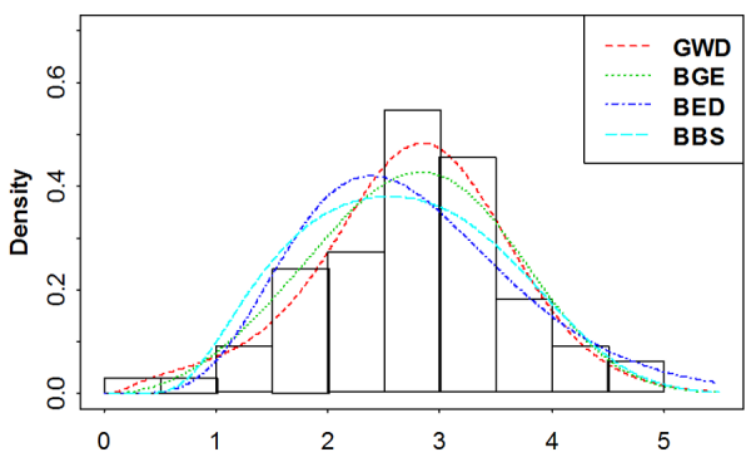

Figure 5. Histogram and the fitted PDFs for the breaking stress of carbon fibers data

Four distributions are used to fit the data: the beta-exponential (BED) defined by Nadarajah and Kotz (2006), the beta-generalized exponential (BGE) defined by Barreto-Souza et al. (2010), the beta-Birnbaum-Saunders, and GWD. The MLEs and goodness of fit statistics are presented in Table 5. The MLEs, standard errors of MLEs, and the AIC statistic of BBS distribution are obtained from Cordeiro and Lemonte (2011). Figure 5 displays the histogram and the PDFs of the fitted distributions.

To compare the four-parameter GWD in (1) to the three-parameter GWD in (11), the likelihood ratio statistic $\eta=14.302$, the Wald statistic $Z_{\hat{\beta}}=2.119$, and the score statistic $S=6.630$ are obtained. All the test statistics lead to rejecting the null hypothesis of three-parameter GWD in favor of the four-parameter GWD at 5\% significance level. The four-parameter GWD outperforms the other three distributions by comparing the AIC and $\mathrm{K}-\mathrm{S}$ statistics. This application suggests that the four-parameter GWD fits unimodal symmetric data very well when compared to the other distributions.

\section{Australian athletes' data}

The Australian athletes' data reported by Cook and Weisberg (1994) contains 13 variables on 102 male and 100 female Australian athletes collected at the Australian Institute of Sport. Jamalizadeh et al. (2011) used the heights for the 100 female athletes and the hemoglobin concentration levels for the 202 athletes to illustrate the application of a generalized skew two-piece skew-normal distribution. Choudhury and Abdul Matin (2011) also used percentage of the hemoglobin blood cell for the male athletes to illustrate the application of an extended skew 


\section{GUMBEL-WEIBULL DISTRIBUTION}

generalized normal distribution. Next, the GWD was applied to two of the variables in the Australian athletes' data, the sum of skin folds (SSF) and the height in centimeters for the 100 female athletes.

The Weibull-Pareto distribution (WPD) defined by Alzaatreh, Famoye and Lee (2013), the beta-normal distribution (BND) defined by Eugene et al. (2002), the exponentiated-Weibull (EWD) defined by Mudholkar and Srivastava (1993), and the GWD are used to fit both data sets.

The sum of skin folds for the 100 female athletes in Table 6 is right skewed (skewness $=0.79$, kurtosis $=0.73$ ). The MLEs and the goodness of fit statistics are in Table 7. The histogram and the densities of the fitted distributions are provided in Figure 6.

Table 6. The sum of skin folds data

\begin{tabular}{cccccccccccc}
\hline 33.8 & 36.8 & 38.2 & 41.1 & 41.6 & 42.3 & 43.5 & 43.5 & 46.1 & 46.2 & 46.3 & 47.5 \\
47.6 & 48.4 & 49.0 & 49.9 & 50.0 & 52.5 & 52.6 & 54.6 & 54.6 & 55.6 & 56.8 & 57.9 \\
58.9 & 59.4 & 61.9 & 62.6 & 62.9 & 65.1 & 67.0 & 68.3 & 68.9 & 69.9 & 70.0 & 71.3 \\
71.6 & 73.9 & 74.7 & 74.9 & 75.1 & 75.2 & 76.2 & 76.8 & 77.0 & 80.1 & 80.3 & 80.3 \\
80.3 & 80.6 & 83.0 & 87.2 & 88.2 & 89.0 & 90.2 & 90.4 & 91.0 & 91.2 & 95.4 & 96.8 \\
97.2 & 97.9 & 98.0 & 98.1 & 98.3 & 98.5 & 99.8 & 99.9 & 101.1 & 102.8 & 102.8 & 103.6 \\
103.6 & 104.6 & 106.9 & 109.0 & 109.1 & 109.5 & 109.6 & 110.2 & 110.7 & 111.1 & 113.5 & 114.0 \\
115.9 & 117.8 & 122.1 & 123.6 & 125.9 & 126.4 & 126.4 & 131.9 & 136.3 & 143.5 & 148.9 & 156.6 \\
156.6 & 171.1 & 181.7 & 200.8 & & & & & & & & \\
\hline
\end{tabular}

The three tests about parameter $\beta$ are used to compare the three- and fourparameter GWD and the tests are not significant. Thus, the three-parameter GWD is adequate to fit the data. On fitting the three-parameter GWD, the K-S statistic shows that it is not as good as the four-parameter GWD. By comparing the goodness of fit statistics among the four distributions in Table 7, it is observed that all distributions provide good fits. The GWD provides the best fit based on the K$\mathrm{S}$ statistic. This application suggests that GWD fits right skewed data very well. 


\section{AL-AQTASH ET AL.}

Table 7. MLEs for sum of skin folds data (standard errors in parentheses)

\begin{tabular}{lcccc} 
Distribution & WPD & BND & EWD & GWD \\
\hline & $\hat{c}=3.6308$ & $\hat{a}=9.7706$ & $\hat{a}=1.2245$ & $\hat{\beta}=1.0299$ \\
& $(1.0568)$ & $(0.4008)$ & $(0.4378)$ & $(1.0094)$ \\
& $\hat{\beta}=0.7183$ & $\hat{b}=0.1967$ & $\hat{\theta}=6.5038$ & $\hat{\sigma}=1.3303$ \\
Parameter & $(0.1799)$ & $(0.0223)$ & $(6.3413)$ & $(0.3218)$ \\
estimates & $\hat{\theta}=23.0497$ & $\hat{\mu}=9.1517$ & $\hat{\sigma}=41.4980$ & $\hat{a}=2.2896$ \\
& $(7.6212)$ & $(4.3612)$ & $(24.4832)$ & $(0.9184)$ \\
& & & & $\hat{\lambda}=81.7841$ \\
& & $\hat{\sigma}=25.4309$ & & $(27.2786)$ \\
\hline Log Likelihood & -486.07 & -487.06 & -487.17 & -485.89 \\
\hline AIC & 978.1 & 982.1 & 980.3 & 979.8 \\
\hline K-S & 0.0825 & 0.0711 & 0.0808 & 0.0705 \\
p-value & 0.5043 & 0.6925 & 0.5307 & 0.7022 \\
\hline
\end{tabular}

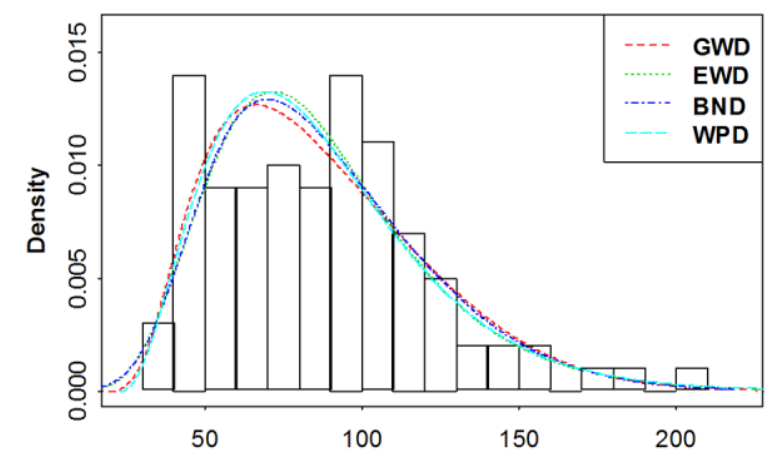

Figure 6. Histogram and the fitted PDFs for sum of skin folds data

The height in centimeters for the 100 Australian female athletes in Table 8 is unimodal and left skewed (skewness $=-0.57$, kurtosis $=1.32$ ). The MLEs and the goodness of fit statistics are in Table 9. The histogram and the PDFs of the fitted distributions are plotted in Figure 7.

The hypothesis in (12) is used to test the significance of the parameter $\beta$. All the tests, the likelihood ratio $(\eta=13.772)$, the Wald $\left(Z_{\hat{\beta}}=2.309\right)$, and the score 


\section{GUMBEL-WEIBULL DISTRIBUTION}

$(S=4.487)$ reject the null hypothesis in favor of the four-parameter GWD. By comparing the goodness of fit statistics among the four distributions it was found that all distributions are competitors as they provide good fits to the data. The fourparameter GWD appears to provide the best p-value of the K-S statistic, and shares the best value of the AIC statistic with the exponentiated-Weibull distribution. This application suggests that GWD fits left skewed unimodal data very well.

Table 8. The heights data

\begin{tabular}{llllllllllll}
\hline 148.9 & 149.0 & 156.0 & 156.9 & 157.9 & 158.9 & 162.0 & 162.0 & 162.5 & 163.0 & 163.9 & 165.0 \\
166.1 & 166.7 & 167.3 & 167.9 & 168.0 & 168.6 & 169.1 & 169.8 & 169.9 & 170.0 & 170.0 & 170.3 \\
170.8 & 171.1 & 171.4 & 171.4 & 171.6 & 171.7 & 172.0 & 172.2 & 172.3 & 172.5 & 172.6 & 172.7 \\
173.0 & 173.3 & 173.3 & 173.5 & 173.6 & 173.7 & 173.8 & 174.0 & 174.0 & 174.0 & 174.1 & 174.1 \\
174.4 & 175.0 & 175.0 & 175.0 & 175.3 & 175.6 & 176.0 & 176.0 & 176.0 & 176.0 & 176.8 & 177.0 \\
177.3 & 177.3 & 177.5 & 177.5 & 177.8 & 177.9 & 178.0 & 178.2 & 178.7 & 178.9 & 179.3 & 179.5 \\
179.6 & 179.6 & 179.7 & 179.7 & 179.8 & 179.9 & 180.2 & 180.2 & 180.5 & 180.5 & 180.9 & 181.0 \\
181.3 & 182.1 & 182.7 & 183.0 & 183.3 & 183.3 & 184.6 & 184.7 & 185.0 & 185.2 & 186.2 & 186.3 \\
188.7 & 189.7 & 193.4 & 195.9 & & & & & & & & \\
\hline
\end{tabular}

Table 9. MLEs for heights data (standard errors in parentheses)

\begin{tabular}{lcccc} 
Distribution & WPD & BND & EWD & GWD \\
\hline & $\hat{c}=8.1823$ & $\hat{a}=0.8697$ & $\hat{a}=14.7405$ & $\hat{\beta}=6.2028$ \\
& $(3.3244)$ & $(0.6607)$ & $(3.2361)$ & $(2.2535)$ \\
& $\hat{\beta}=2.8449$ & $\hat{b}=6.3120$ & $\hat{\theta}=2.7836$ & $\hat{\sigma}=3.9326$ \\
Parameter & $(1.1079)$ & $(0.7454)$ & $(1.3390)$ & $(2.9597)$ \\
estimates & $\hat{\theta}=125.1377$ & $\hat{\mu}=191.46$ & $\hat{\sigma}=170.26$ & $\hat{a}=12.268$ \\
& $(16.9742)$ & $(3.9729)$ & $(4.5462)$ & $(2.1530)$ \\
& & & & $\hat{\lambda}=147.02$ \\
& & $\hat{\sigma}=12.0376$ & & $(12.5835)$ \\
\hline Log Likelihood & -351.03 & -350.31 & -350.37 & -349.33 \\
\hline AIC & 708.1 & 708.6 & 706.7 & 706.7 \\
\hline K-S & 0.0801 & 0.0725 & 0.0711 & 0.0565 \\
p-value & 0.5427 & 0.6692 & 0.6923 & 0.9071 \\
\hline
\end{tabular}


AL-AQTASH ET AL.

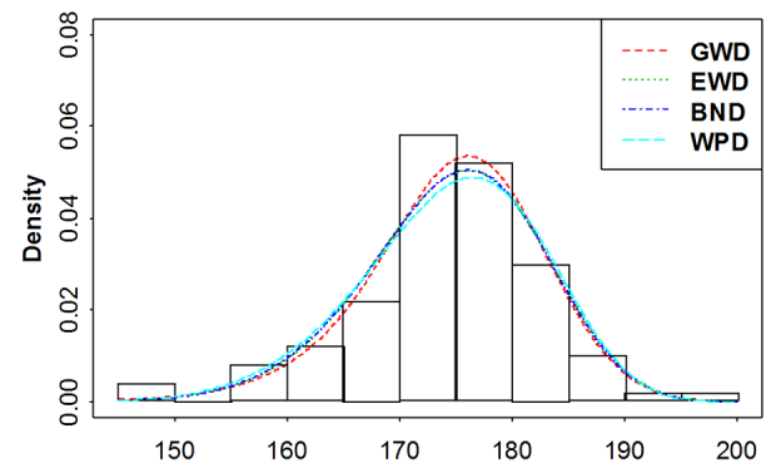

Figure 7. Histogram and the fitted PDFs for heights data

\section{Application to lifetime data}

Two data sets that represent the stress rapture life in hours of Kevlar 49/epoxy strands when subjected to a constant sustained stress level pressure until failure are used to illustrate an application to lifetime data. Both data sets are from Andrews and Herzberg (1985) and the original source is Barlow et al. (1984). Cooray and Ananda (2008) used the data sets to illustrate the usefulness of the generalized half normal distribution (GHN) when compared to other commonly used distributions. Four distributions are used to fit both data sets: the exponentiated-Weibull, betanormal, generalized half normal, and GWD. The MLEs, log-likelihood value, and the value of K-S statistic of GHN distribution are obtained from Cooray and Ananda (2008).

The data set in Table 10 represents the failure times of Kevlar 49/epoxy strands when the pressure is at $90 \%$ stress level. This data is leptokurtic, unimodal, highly right skewed, and has reversed-J shape with a potential outlier (skewness $=$ 3.05 , kurtosis $=14.47$ ).

The MLEs, $\log$ likelihood values, values of AIC and K-S, and p-values of the K-S are in Table 11. Figure 8 contains the histogram and the PDFs of the fitted distributions. The three tests about parameter $\beta$ are used to test its significance. Only the likelihood ratio test rejects the null hypothesis of the three-parameter GWD, while the other two tests fail to reject the null hypothesis in (12). Because the likelihood ratio test is the most powerful when $\beta>1$, one can conclude that the parameter $\beta$ is significant. 


\section{GUMBEL-WEIBULL DISTRIBUTION}

Table 10. Kevlar 49/epoxy strands failure times data (pressure at $90 \%$ )

\begin{tabular}{lllllllllll}
\hline 0.01 & 0.01 & 0.02 & 0.02 & 0.02 & 0.03 & 0.03 & 0.04 & 0.05 & 0.06 & 0.07 \\
0.07 & 0.08 & 0.09 & 0.09 & 0.10 & 0.10 & 0.11 & 0.11 & 0.12 & 0.13 & 0.18 \\
0.19 & 0.20 & 0.23 & 0.24 & 0.24 & 0.29 & 0.34 & 0.35 & 0.36 & 0.38 & 0.40 \\
0.42 & 0.43 & 0.52 & 0.54 & 0.56 & 0.60 & 0.60 & 0.63 & 0.65 & 0.67 & 0.68 \\
0.72 & 0.72 & 0.72 & 0.73 & 0.79 & 0.79 & 0.80 & 0.80 & 0.83 & 0.85 & 0.90 \\
0.92 & 0.95 & 0.99 & 1.00 & 1.01 & 1.02 & 1.03 & 1.05 & 1.10 & 1.10 & 1.11 \\
1.15 & 1.18 & 1.20 & 1.29 & 1.31 & 1.33 & 1.34 & 1.40 & 1.43 & 1.45 & 1.50 \\
1.51 & 1.52 & 1.53 & 1.54 & 1.54 & 1.55 & 1.58 & 1.60 & 1.63 & 1.64 & 1.80 \\
1.80 & 1.81 & 2.02 & 2.05 & 2.14 & 2.17 & 2.33 & 3.03 & 3.03 & 3.34 & 4.20 \\
4.69 & 7.89 & & & & & & & & & \\
\hline
\end{tabular}

Table 11. MLEs for Kevlar 49/epoxy strands failure times data (pressure at 90\%) (standard errors in parentheses)

\begin{tabular}{lcccc} 
Distribution & \multicolumn{1}{c}{ GHN $^{*}$} & EWD & BND & GWD \\
\hline & $\hat{\theta}=1.2238$ & $\hat{a}=1.0604$ & $\hat{a}=12.4298$ & $\hat{\beta}=1.8064$ \\
& & $(0.2399)$ & $(0.3071)$ & $(0.5037)$ \\
& $\hat{\alpha}=0.7108$ & $\hat{\theta}=0.7929$ & $\hat{b}=0.4467$ & $\hat{\sigma}=3.2713$ \\
Parameter & $(0.2870)$ & $(0.0475)$ & $(0.6459)$ \\
estimates & $\hat{\sigma}=1.2180$ & $\hat{\mu}=-1.5065$ & $\hat{a}=0.9200$ \\
& & $(0.3933)$ & $(0.1347)$ & $(0.1594)$ \\
& & & $\hat{\sigma}=1.1413$ & $\hat{\lambda}=0.2071$ \\
& & & $(0.0317)$ & $(0.1072)$ \\
\hline Log Likelihood & -103.33 & -102.79 & -132.98 & -100.23 \\
\hline AIC & 210.7 & 211.6 & 274.0 & 208.5 \\
\hline K-S & 0.0800 & 0.0844 & 0.1129 & 0.0687 \\
p-value & 0.5377 & 0.4680 & 0.1526 & 0.7266 \\
\hline
\end{tabular}

*MLEs, log likelihood, and K-S from Cooray and Ananda (2008), the standard errors were not given.

By comparing the p-value of the K-S and the AIC among the four distributions, it is observed that the GWD outperforms the other distributions. This application suggests that the GWD fits unimodal reversed-J shape data very well. 
AL-AQTASH ET AL.

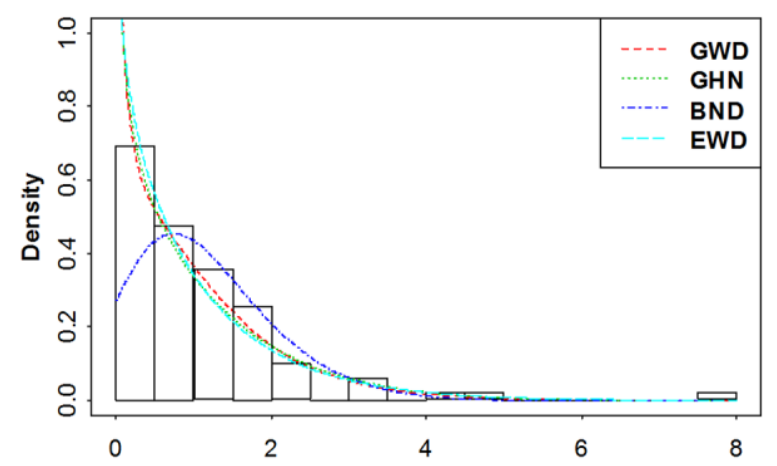

Figure 8. Histogram and the fitted PDFs for failure times data (90\% pressure)

The second part of the Kevlar data in Table 12 represent the failure times when the pressure is at $70 \%$ stress level. This data is multimodal, platykurtic, and approximately symmetric (skewness $=0.1$, kurtosis $=-0.79$ ) with very wide range. Table 13 contains the MLEs, AIC and K-S statistics, and the p-value of the K-S statistic. Figure 9 provides the histogram and the PDFs of the fitted distributions.

Table 12. Kevlar 49/epoxy strands failure times data (pressure at $70 \%$ )

\begin{tabular}{cccccccccc}
\hline 1051 & 1337 & 1389 & 1921 & 1942 & 2322 & 3629 & 4006 & 4012 & 4063 \\
4921 & 5445 & 5620 & 5817 & 5905 & 5956 & 6068 & 6121 & 6473 & 7501 \\
7886 & 8108 & 8546 & 8666 & 8831 & 9106 & 9711 & 9806 & 10205 & 10396 \\
10861 & 11026 & 11214 & 11362 & 11604 & 11608 & 11745 & 11762 & 11895 & 12044 \\
13520 & 13670 & 14110 & 14496 & 15395 & 16179 & 17092 & 17568 & 17568 & \\
\hline
\end{tabular}

When the three tests about $\beta$ are used to compare the three-parameter GWD with the four-parameter GWD, the parameter $\beta$ is not significantly different from 1. The three-parameter GWD was also fitted to the data and the result is comparable to that of four-parameter GWD. By using the K-S statistic, the three-parameter GWD is not as good as the four-parameter GWD.

By comparing the goodness of fit statistics in Table 13 among the four distributions, it was found that the four distributions are competitors and fit the data very well. The GWD and the beta-normal distribution capture the data with a bimodal density curve. The GHN with the smallest AIC and largest p-value of the $\mathrm{K}-\mathrm{S}$ statistic does not capture the bimodality property. This application suggests that GWD is capable of providing an adequate fit to bimodal data. 


\section{GUMBEL-WEIBULL DISTRIBUTION}

Table 13. MLEs for Kevlar 49/epoxy strands failure times data (pressure at $70 \%$ ) (standard errors in parentheses)

\begin{tabular}{lcccc} 
Distribution & GHN $^{*}$ & EWD & BND & GWD \\
\hline & $\hat{\theta}=10906.98$ & $\hat{a}=5.2226$ & $\hat{a}=0.1626$ & $\hat{\beta}=1.3118$ \\
& & $(2.9883)$ & $(0.1039)$ & $(0.5144)$ \\
& $\hat{\alpha}=1.64067$ & $\hat{\theta}=0.2644$ & $\hat{b}=0.1157$ & $\hat{\sigma}=4.1091$ \\
Parameter & & $(0.1887)$ & $(0.0199)$ & $(1.0456)$ \\
estimates & $\hat{\sigma}=14559$ & $\hat{\mu}=7826$ & $\hat{a}=2.6948$ \\
& & $(2007.54)$ & $(1759.97)$ & $(0.8101)$ \\
& & & $\hat{\sigma}=1339.35$ & $\hat{\lambda}=6165.69$ \\
& & & $(245.62)$ & $(1749.51)$ \\
\hline Log Likelihood & -479.56 & -479.03 & -480.52 & -478.51 \\
\hline AIC & 963.1 & 964.1 & 969.0 & 965.0 \\
\hline K-S & 0.067 & 0.0825 & 0.0797 & 0.0749 \\
p-value & 0.9804 & 0.8926 & 0.9144 & 0.9462 \\
\hline
\end{tabular}

* MLEs, log likelihood, and K-S from Cooray and Ananda (2008), the standard errors were not given.

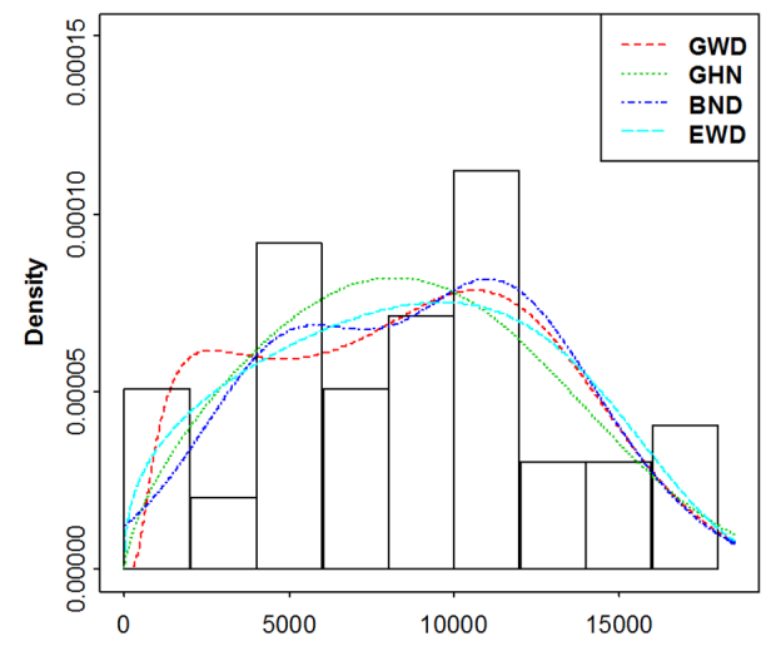

Figure 9. Histogram and the fitted PDFs for failure times data (70\% pressure) 


\section{AL-AQTASH ET AL.}

\section{Summary and conclusion}

In this article, some properties of the four-parameter GWD, a member of the $T-X$ family of distributions are provided. The paper provides important properties such as modes and mean deviations for GWD. The method of maximum likelihood is proposed for parameter estimation. The result of the simulation on the performance of the maximum likelihood method shows that the method is appropriate for estimating the parameters of GWD. Three test statistics are used to test the significance of the parameter $\beta$. The score test is the most powerful when $\beta \leq 1$, while the likelihood ratio test is the most powerful when $\beta>1$. Five datasets are used to illustrate the application of the GWD and the results are compared to other existing distributions. The results of these applications suggest that GWD can be used to fit right-skewed, left-skewed, symmetric, unimodal and bimodal data.

\section{References}

Al-Aqtash, R., Famoye, F. and Lee, C. (2014). On generating a new family of distributions using the logit function. To appear in the Journal of Probability and Statistical Science.

Alzaatreh, A., Famoye, F. \& Lee, C. (2013). Weibull-Pareto distribution and its applications, Communications in Statistics - Theory and Methods, 42, 16731691.

Alzaatreh, A., Lee, C. \& Famoye, F. (2013). A new method for generating families of continuous distributions, Metron, 71(1), 63-79.

Andrews, D. F. \& Herzberg, A. M. (1985). Data: A Collection of Problems from Many Fields for the Student and Research Worker (Springer Series in Statistics). New York: Springer.

Barlow, R. E., Toland, R. H. \& Freeman, T. (1984). A Bayesian analysis of stress-rupture life of Kevlar 49/epoxy spherical pressure vessels. In T. D. Dwivedi (Ed.), Proceedings of the Canadian Conference in Applied Statistics. New York: Marcel Dekker.

Barreto-Souza, W., Santos, A. H. S. \& Cordeiro, G. M. (2010). The beta generalized exponential distribution, Journal of Statistical Computation and Simulation, 80(2), 159-172.

Beirlant, J., Goegebeur, Y., Segers, J. \& Teugels, J. (2006). Statistics of Extremes: Theory and Applications. West Sussex, England: John Wiley and Sons Ltd. 


\section{GUMBEL-WEIBULL DISTRIBUTION}

Birnbaum, Z. W. \& Saunders, S. C. (1969). A new family of life distributions. Journal of Applied Probability, 6, 319-327.

Choudhury, K. \& Abdul Matin, M. (2011). Extended skew generalized normal distribution. Metron, 69(3), 265-278.

Cook, R. D. \& Weisberg, S. (1994). An Introduction to Regression Graphics. New York: John Wiley and Sons, Inc.

Cooray, K. \& Ananda, M. (2008). A generalization of the half-normal distribution with applications to lifetime data. Communications in Statistics Theory and Methods, 37, 1323-1337.

Cordeiro, G. M. \& Lemonte, A. J. (2011). The $\beta$-Birnbaum-Saunders distribution: An improved distribution for fatigue life modeling. Computational Statistics and Data Analysis, 55(3), 1445-1461.

Eugene, N., Lee, C. \& Famoye, F. (2002). Beta-normal distribution and its applications. Communications in Statistics - Theory and Methods, 31(4), 497512.

Gumbel, E. J. (1958). Statistics of Extremes. New York: Columbia University Press.

Jamalizadeh, A., Arabpour, A.R., \& Balakrishnan, N. (2011). A generalized skew two-piece skew-normal distribution. Statistical Papers, 52(2), 431-446.

Johnson, N. L., Kotz, S. \& Balakrishnan, N. (1994). Continuous Univariate Distributions, Vol. 1 (2nd ed.). New York: John Wiley and Sons, Inc.

Johnson, N. L., Kotz, S. \& Balakrishnan, N. (1995). Continuous Univariate Distributions, Vol. 2 (2nd ed.). New York: John Wiley and Sons, Inc.

Kotz, S. \& Nadarajah, S. (2000). Extreme Value Distributions. London: Imperial College Press.

Mudholkar, G. S. \& Srivastava, D. K. (1993). Exponentiated Weibull family for analyzing bathtub failure-rate data. IEEE Transactions on Reliability, 42(2), 299-302.

Nadarajah, S. \& Kotz, S. (2006). The beta exponential distribution, Reliability Engineering and System Safety, 91(6), 689-697.

Neyman, J. \& Pearson, E. S. (1928). On the use and interpretation of certain test criteria for purposes of statistical inference. Biometrika, 20, 175-240, 263294.

Nicholas, M. D. \& Padgett, W. J. (2006). A bootstrap control for Weibull percentiles. Quality and Reliability Engineering International, 22, 141-151. 


\section{AL-AQTASH ET AL.}

Rao. C. R. (1948). Large sample tests of statistical hypotheses concerning several parameters with application to problems of estimation. Proceedings of the Cambridge Philosophical Society, 44, 50-57.

Wald, A. (1943). Tests of statistical hypotheses concerning several parameters when the number of observations is large. Transactions of the American Mathematical Society, 54(3), 426-482. 